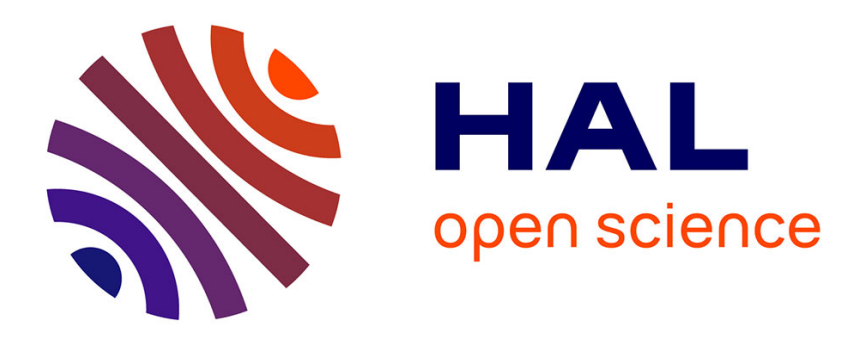

\title{
Locking materials and the topology of optimal shapes
}

Frederic Golay, Pierre Seppecher

\section{To cite this version:}

Frederic Golay, Pierre Seppecher. Locking materials and the topology of optimal shapes. European Journal of Mechanics - A/Solids, 2001, 20 (4), pp.631-644. hal-00528969

\section{HAL Id: hal-00528969 \\ https://hal.science/hal-00528969}

Submitted on 23 Oct 2010

HAL is a multi-disciplinary open access archive for the deposit and dissemination of scientific research documents, whether they are published or not. The documents may come from teaching and research institutions in France or abroad, or from public or private research centers.
L'archive ouverte pluridisciplinaire HAL, est destinée au dépôt et à la diffusion de documents scientifiques de niveau recherche, publiés ou non, émanant des établissements d'enseignement et de recherche français ou étrangers, des laboratoires publics ou privés. 


\title{
Locking materials and the topology of optimal shapes
}

\author{
Frédéric Golay *, Pierre Seppecher \\ Laboratoire d'analyse non-linéaire appliquée et modélisation (ANAM), université de Toulon et du Var, \\ 83957 La Garde cedex, France
}

(Received 24 July 2000; revised and accepted 21 February 2001)

\begin{abstract}
We study a problem of structural optimisation using the fictitious material approach. This is connected with the equilibrium of locking materials, which can be approximated by strongly non linear materials. A finite element simulation allows us to experiment with some conjectures about the topology of the optimal solutions. ( 2001 Éditions scientifiques et médicales Elsevier SAS

shape optimisation / locking material / topological optimisation / fictitious material / finite elements
\end{abstract}

\section{Introduction}

Structural optimisation is a major objective in the conception of industrial systems. In particular, for a given desired performance, engineers may need to minimise the mass of a structure by using an adapted geometry. Early methods consist of progressively tuning some geometric parameters of a postulated initial shape. The optimal design obtained in this way depends strongly on the initial one: the optimisation process cannot change the fundamental topological characteristics of the shape. Methods which allow such modifications (Allaire et al., 1997; Bendsoe, 1995) are called topological optimisation. Here we consider a structure submitted to a given load and we try to get a structure with the smallest volume for a desired compliance (i.e. global stiffness, or stored elastic energy for the given load). It is now well known that this problem may admit no solution in the classical sense: the optimal 'shape' may consist of an intricate mixture of material and holes. The optimal solution has to be understood in the framework of homogenisation theory and the optimisation problem has to be set at the very beginning in a relaxed form. However, as the set of all possible effective stiffness tensors for a given volume fraction of the material is rather intricate (Allaire and Kohn, 1993; Aubry, 1999; Bendsoe, 1995), it seems difficult to find analytic solutions or mathematical properties for optimal designs. That is why we consider a very similar but much simpler problem: we assume that the stiffness tensor depends linearly on the volume fraction (or the mass density) of the material. In 3-D this assumption is not realistic as the stiffness of a composite material with a given volume fraction is weaker than the stiffness obtained assuming a linear dependence: the problem we consider is then called 'fictitious material optimisation'. In 2-D, the problem corresponds to the optimisation of the thickness of a plate submitted to a plane load (plane stress). These linear dependence or different 'artificial mixture' rules are discussed in (Duysinx, 1996).

The optimal designs obtained using homogenisation theory or the fictitious approach are qualitatively similar. Hopefully our results could then be extended to the optimal structures obtained by the homogenisation method.

We show that the problem of fictitious material optimisation is equivalent to the equilibrium problem of a perfect locking material (i.e. a material whose strain tensor must belong to a given bounded set, in this case its

\footnotetext{
* Correspondence and reprints.

E-mail address: golay@isitv.univ-tln.fr (F. Golay).
} 
internal energy density vanishes). This convex minimisation enables us to give non-trivial analytical solutions (section 4.1). Its regularisation leads to a simple non-linear elasticity problem (section 3). Some 2-D numerical solutions are presented (section 5) and compared to analytical solutions. The numerical solutions enable us to test some conjectures about the topology of optimal solutions. In the case when the support of the optimal design is a connected set, it seems that this support is a simply-connected bounded set. In particular, we can prove that circular holes cannot be present in an optimal design.

This conjecture, if confirmed, is remarkable: the topological complexity is not introduced by the optimisation process, but only by a later penalisation process (such a penalisation is generally used to get out of the relaxed formulation and to obtain a classical solution (Allaire et al., 1997). Only this penalisation process justifies the name of 'topological optimisation' for the whole process.

\section{Optimal design of membranes}

\subsection{Mathematical formulation}

Let us consider a thin plane membrane of variable thickness $h(x), x$ belonging to some bounded domain $\Omega \subset \mathbf{R}^{2}$. Its volume $\mathcal{V}$ is then given by:

$$
\mathcal{V}=\int_{\Omega} h(x) \mathrm{d} s
$$

Assuming that the membrane is made of an homogeneous isotropic elastic material with Young's modulus $E$ and Poisson coefficient $v$, its elastic energy under plane stress conditions is:

$$
\int_{\Omega} \frac{1}{2} \varepsilon_{v}(x): D: \varepsilon_{v}(x) h(x) \mathrm{d} s,
$$

where $v \in H^{1}\left(\Omega, \mathbf{R}^{2}\right)$ denotes the displacement field of the membrane, $\varepsilon_{v}$ the plane strain tensor $\varepsilon_{v}=$ $\frac{1}{2}\left(\nabla v+\nabla^{t} v\right)$ and $D$ is the two-dimensional fourth-order stiffness tensor:

$$
D_{i j k l}=\frac{E v}{1-v^{2}} \delta_{i j} \delta_{k l}+\frac{E}{1+v} \delta_{i k} \delta_{j l} .
$$

At equilibrium the displacement field $u$ minimises the potential energy:

$$
J(v, h)=\int_{\Omega} \frac{1}{2} \varepsilon_{v}: D: \varepsilon_{v} h \mathrm{~d} s-\int_{\mathcal{L}} F \cdot v \mathrm{~d} \ell,
$$

where $F$ denotes a line density of applied forces along a line $\mathcal{L}$. Other types of measures could be considered for $F$, but, for the sake of simplicity, this will not be done here. For equilibrium to be possible, we assume that the resulting force and moment vanish $\left(\int_{\mathcal{L}} F \mathrm{~d} \ell=\int_{\mathcal{L}} x \wedge F \mathrm{~d} \ell=0\right)$. Such conditions could be replaced by Dirichlet conditions for the displacement on some part of $\Omega$.

The elastic energy at equilibrium can be rewritten, in terms of the equilibrium displacement field $u$,

$$
\mathcal{E}(h)=-\inf _{v} J(v, h)=-J(u, h)=\int_{\Omega} \frac{1}{2} \varepsilon_{u}: D: \varepsilon_{u} h \mathrm{~d} s=\frac{1}{2} \int_{\mathcal{L}} F \cdot u \mathrm{~d} \ell .
$$

Assume that we need to maximise the global stiffness of the structure (or which is equivalent, to minimise the volume of the structure) for the given single loading $F$. An optimal design of the membrane is a function 
$h(x)$ which, for a given volume $\mathcal{V}$, minimises the equilibrium elastic energy. The optimisation problem reads:

$$
E=\inf _{h}\left\{\mathcal{E}(h) ; h(x) \geqslant 0 ; \int_{\Omega} h(x) \mathrm{d} s=\mathcal{V}\right\}
$$

or, using (3) and (4):

$$
E=-\sup _{h} \inf _{v}\left\{\int_{\Omega} \frac{1}{2} \varepsilon_{v}: D: \varepsilon_{v} h \mathrm{~d} s-\int_{\mathcal{L}} F \cdot v \mathrm{~d} \ell ; h \geqslant 0 ; \int_{\Omega} h \mathrm{~d} s=\mathcal{V}\right\} .
$$

Let us remark that, even in this relaxed formulation, the existence of an optimal design (a function $h$ minimising (6)) is generally not assured. For some special loading $F$ the optimal solution may concentrate along a line: indeed, as proved in (Bouchitté and Buttazzo, 2001), the optimal solution $h$ has to be sought in the space of measures. Examples of this phenomenon are given in (Bouchitté et al., 1997-a) for conductivity optimisation and can easily be extended to our problem. In this paper we assume that forces are such that $h$ and the associated displacement field $u$ exist and are sufficiently regular.

Note that the line $\mathcal{L}$, where external forces are applied, may be different from the boundary $\partial \Omega$ of $\Omega$ and may not even coincide with a part of the boundary. This allows us to consider the case $\Omega=\mathbf{R}^{2}$ or, in numerical studies, to consider a domain much larger than the support of applied forces. Then we can obtain optimal structures which are not subjected to any geometrical constraint.

\subsection{Perfect locking materials}

We show now that the problem (6) is equivalent to the resolution of the equilibrium of a perfect locking material. We search, a priori, the supremum in (6) in the convex compact set $\mathcal{M}$ of all non-negative measures $h$ on $\bar{\Omega}$ which verify $\int h=\mathcal{V}$. By a density argument, the infimum can be sought in $C^{\infty}(\bar{\Omega})$, so (6) can be rewritten:

$$
E=\inf _{h \in \mathcal{M}} \sup _{v \in C^{\infty}(\bar{\Omega})}\{-J(v, h)\}
$$

As, on $C^{\infty}(\bar{\Omega}) \times \mathcal{M},-J$ is concave with respect to the first variable and convex (linear) lower semicontinuous with respect to the second one, the 'lop sided minimax theorem' (cf. chapter 6 of (Aubin and Ekeland, 1984)) allows us to interchange inf and sup in (7):

$$
E=\sup _{v \in C^{\infty}(\bar{\Omega})} \inf _{h \in \mathcal{M}}\{-J(v, h)\}=-\inf _{v \in C^{\infty}(\bar{\Omega})} \sup _{h \in \mathcal{M}} J(v, h) .
$$

This last supremum in $h$ is easy to compute (it is enough to concentrate $h$ where $\left\|\varepsilon_{v}: D: \varepsilon_{v}\right\|_{\infty}$ reaches its maximum), and we get:

$$
E=-\inf _{v \in C^{\infty}(\bar{\Omega})}\left\{-\int_{\mathcal{L}} F \cdot v \mathrm{~d} \ell+\left\|\varepsilon_{v}: D: \varepsilon_{v}\right\|_{\infty} \frac{\mathcal{V}}{2}\right\}
$$

In order to get rid of the influence of the total volume $\mathcal{V}$, we can consider, for each $v \in C^{\infty}(\bar{\Omega})$, the quantities $s=\left\|\varepsilon_{v}: D: \varepsilon_{v}\right\|_{\infty}^{1 / 2}$ and $w=s^{-1} v$ and write (9) as:

$$
E=-\inf _{w \in C^{\infty}(\bar{\Omega}), s \geqslant 0}\left\{-s \int_{\mathcal{L}} F \cdot w \mathrm{~d} \ell+\frac{\mathcal{V} s^{2}}{2},\left\|\varepsilon_{v}: D: \varepsilon_{v}\right\|_{\infty}=1\right\} .
$$


As the minimisation in $s$ is straightforward, we obtain:

$$
E=-\frac{1}{2 \mathcal{V}}\left[\inf _{w}\left\{\int_{\mathcal{L}}-F \cdot w \mathrm{~d} \ell ;\left\|\varepsilon_{w}: D: \varepsilon_{w}\right\|_{\infty}=1\right\}\right]^{2}
$$

or, in an equivalent way,

$$
E=-\frac{1}{2 \mathcal{V}}\left[\inf _{w}\left\{\int_{\mathcal{L}}-F \cdot w \mathrm{~d} \ell ;\left\|\varepsilon_{w}: D: \varepsilon_{w}\right\|_{\infty} \leqslant 1\right\}\right]^{2}
$$

In this last formulation, the minimisation problem corresponds to the equilibrium of a perfect locking material (Demengel and Suquet, 1986): the strain tensor of such a material has to satisfy the constraint $\left\|\varepsilon_{w}: D: \varepsilon_{w}\right\|_{\infty} \leqslant 1$, then its volume energy vanishes.

These results also hold in the more general case when the elastic material is a non-linear one. This has been proved by G. Bouchitté and G. Buttazzo (2001).

Note that formulation (12) can be obtained by using the stress based formulation of (Allaire and Kohn, 1993). In our simpler case the set of all stiffness tensors for a given thickness $h$, is reduced to the singleton $h D$. Then, the variational problem (2.16) of (Allaire and Kohn, 1993) is easily explicated: in our case this problem becomes convex and corresponds to the dual formulation of (12).

A similar formulation has already been used to study optimal truss structures (Michell, 1904; Lagache, 1981; Rozvany, 1989). Indeed, problems (P2) or (P3) in (Lagache, 1981) clearly correspond to the equilibrium of a perfect locking material. In the case of optimal trusses, the strain tensor has to verify the constraint $\left\|\left|\varepsilon_{w}\right|\right\| \leqslant 1$ (where $\||\varepsilon|||$ denotes the highest singular value of $\varepsilon$ ) while in our case the constraint is $\left\|\varepsilon_{w}: D: \varepsilon_{w}\right\|_{\infty} \leqslant 1$. The locking material formulation for optimal trusses has been particularly fruitful and optimal solutions can be described in terms of Hencky nets (Strang and Kohn, 1983). Here we investigate how the results obtained for optimal trusses can be extended to the problem (12). We cannot obtain a simple structure like Hencky nets, nevertheless the formulation (12) enables us to get some analytical solutions and some topological properties of optimal designs.

Formulation (9) has already been used in finite dimension by (Ben-Tal and Bendsoe, 1993) for the numerical analysis of optimal trusses and by (Zowe et al., 1997) for the finite element study of fictitious material. We will first regularise our convex minimisation problems before discretising them.

\section{Numerical formulation}

\subsection{Problem regularisation}

In order to solve numerically (9) or (12), let us regularise the term containing the norm $\|\cdot\|_{\infty}$.

In the first formulation, we approximate the infinity norm by a $p$-norm where $p$ is a large parameter. Indeed:

$$
\left\|\varepsilon_{v}: D: \varepsilon_{v}\right\|_{\infty}=\lim _{p \rightarrow \infty}\left\|\varepsilon_{v}: D: \varepsilon_{v}\right\|_{p}=\lim _{p \rightarrow \infty}\left[\int_{\Omega}\left(\varepsilon_{v}: D: \varepsilon_{v}\right)^{p} \mathrm{~d} s\right]^{1 / p} .
$$

Then we deal with the following optimisation problem:

$$
\lim _{p \rightarrow \infty}\left(\inf _{v} J_{p}(v)\right)
$$


where

$$
J_{p}(v):=\mathcal{V}\left(\int_{\Omega}\left(\varepsilon_{v}: D: \varepsilon_{v}\right)^{p} \mathrm{~d} s\right)^{1 / p}-2 \int_{\mathcal{L}} F \cdot v \mathrm{~d} \ell .
$$

As the derivative of $J_{p}$ is given by:

$$
J_{p}^{\prime}(u) \cdot v=\mathcal{V}\left(\int_{\Omega}\left(\varepsilon_{u}: D: \varepsilon_{u}\right)^{p} \mathrm{~d} s\right)^{1 / p-1}\left(\int_{\Omega} 2\left(\varepsilon_{u}: D: \varepsilon_{u}\right)^{p-1}\left(\varepsilon_{u}: D: \varepsilon_{v}\right) \mathrm{d} s\right)-2 \int_{\mathcal{L}} F \cdot v \mathrm{~d} \ell,
$$

the variational formulation of the optimisation problem becomes:

$$
\int_{\Omega} H(u) \varepsilon_{u}: D: \varepsilon_{v} \mathrm{~d} s=\int_{\mathcal{L}} F \cdot v \mathrm{~d} \ell
$$

where

$$
H(u):=\mathcal{V}\left(\varepsilon_{u}: D: \varepsilon_{u}\right)^{p-1}\left(\int_{\Omega}\left(\varepsilon_{u}: D: \varepsilon_{u}\right)^{p} \mathrm{~d} s\right)^{1 / p-1} .
$$

This variational formulation corresponds to a non-local elastic problem. Indeed the stiffness tensor $H D$, through $H$, depends on the solution $u$ on the whole domain.

For a given $H$, this formulation corresponds again to the equilibrium of an elastic plate of thickness $H$ under plane stress. The comparison of (17) and (4) shows that $H$ corresponds to the optimal design $h$ we are looking for. Here $H$ depends on the global strain energy.

In the second formulation (12), introducing the indicator function defined on $\mathbf{R}^{+}$by:

$$
I(t):= \begin{cases}\infty & \text { if } t>1, \\ 0 & \text { otherwise }\end{cases}
$$

the optimisation problem becomes:

$$
\inf _{w}\left\{-\int_{\mathcal{L}} F \cdot w \mathrm{~d} \ell+\int_{\Omega} I\left(\varepsilon_{w}: D: \varepsilon_{w}\right) \mathrm{d} s\right\}
$$

Let us now approximate the indicator function $I$ by the function $I_{p}$, defined by $I_{p}(t):=(2 p)^{-1} t^{p}, p$ being a large parameter. The minimisation problem becomes:

$$
\lim _{p \rightarrow \infty}\left(\inf _{w} \tilde{J}_{p}(w)\right)
$$

where

$$
\tilde{J}_{p}(w):=\int_{\Omega} \frac{1}{2 p}\left(\varepsilon_{w}: D: \varepsilon_{w}\right)^{p} \mathrm{~d} s-\int_{\mathcal{L}} F \cdot w \mathrm{~d} \ell .
$$

As the derivative of $\tilde{J}_{p}$ is given by:

$$
\tilde{J}_{p}^{\prime}(u) \cdot v=\left(\int_{\Omega}\left(\varepsilon_{u}: D: \varepsilon_{u}\right)^{p-1}\left(\varepsilon_{u}: D: \varepsilon_{v}\right) \mathrm{d} s\right)-\int_{\mathcal{L}} F \cdot v \mathrm{~d} \ell,
$$


the variational formulation of the optimisation problem is written again:

$$
\int_{\Omega} \tilde{H}(u) \varepsilon_{u}: D: \varepsilon_{v} \mathrm{~d} s=\int_{\mathcal{L}} F \cdot v \mathrm{~d} \ell
$$

where

$$
\tilde{H}(u):=\left(\varepsilon_{u}: D: \varepsilon_{u}\right)^{p-1} .
$$

This variational formulation corresponds to a simple local elastic problem. Again the comparison of (23) and (4) shows that $\tilde{H}$ corresponds to the optimal design $h$ we are looking for. Here $\tilde{H}$ depends simply on the local strain energy density. Note that the problems (16)-(17) and (23)-(24) are equivalent up to a multiplicative constant. They are both non linear, but convex, elastic equilibrium problems. They lead to the same optimal design.

Similar formulations have been established by Ben-Tal and Bendsoe (1993) for the study of optimal trusses or by Petersson (1999) for the study (very closely connected to ours) of optimal variable thickness sheets.

\subsection{Finite element formulation}

We use a finite element formulation (Batoz and Dhatt, 1995) for our problems. Let us denote $\{u\}$ the vector of the nodal unknowns and, for each element $e, N^{e}$ the interpolation functions. Then the displacement field is approximated on $e$ by:

$$
u(x)=\left[N^{e}(x)\right]\{u\},
$$

which, by differentiation, defines $B^{e}$ such that:

$$
\varepsilon_{u}(x)=\left[B^{e}(x)\right]\{u\} .
$$

Then we write on each element $e$ :

$$
\varepsilon_{u}: D: \varepsilon_{v}=\{u\}^{T}\left[B^{e}\right]^{T}[D]\left[B^{e}\right]\{v\}=\{u\}^{T}\left[K^{e}\right]\{v\} .
$$

Here $\left[K^{e}\right]:=\left[B^{e}\right]^{T}[D]\left[B^{e}\right]$ is the elementary elastic stiffness matrix. Decomposing the domain $\Omega$ with the supports $V^{e}$ of the finite element and denoting:

$$
E_{p}:=\int_{\Omega}\left(\varepsilon_{u}: D: \varepsilon_{u}\right)^{p} \mathrm{~d} s=\sum_{e} \int_{V^{e}}\left(\{u\}^{T}\left[K^{e}\right]\{u\}\right)^{p} \mathrm{~d} s,
$$

the finite element formulation of (16) is written as:

$$
\mathcal{V} E_{p}^{1 / p-1} \sum_{e} \int_{V^{e}}\left(\{u\}^{T}\left[K^{e}\right]\{u\}\right)^{p-1}\{v\}^{T}\left[K^{e}\right]\{u\} \mathrm{d} s=\sum_{e} \int_{\partial V^{e}}\{v\}^{T}\left[N^{e}\right]\{F\} \mathrm{d} \ell .
$$

As this equality holds for any $\{v\}$, the residue:

$$
\begin{aligned}
\left\{R_{p}\right\}(\{u\}):= & -\sum_{e} \int_{\partial V^{e}}\left[N^{e}\right]\{F\} \mathrm{d} \ell+\mathcal{V}\left(\sum_{e} \int_{V^{e}}\left(\{u\}^{T}\left[K^{e}\right]\{u\}\right)^{p} \mathrm{~d} s\right)^{1 / p-1} \\
& \times \sum_{e} \int_{V^{e}}\left(\{u\}^{T}\left[K^{e}\right]\{u\}\right)^{p-1}\left[K^{e}\right]\{u\} \mathrm{d} s,
\end{aligned}
$$


must vanish.

In the same way the residue of the finite element formulation of (23) becomes:

$$
\left\{\tilde{R}_{p}\right\}(\{u\}):=\sum_{e} \int_{V^{e}}\left(\{u\}^{T}\left[K^{e}\right]\{u\}\right)^{p-1}\left[K^{e}\right]\{u\} \mathrm{d} s-\sum_{e} \int_{\partial V^{e}}\left[N^{e}\right]\{F\} \mathrm{d} \ell .
$$

\subsection{Computational method}

As, for a given value of $p \neq 1$, the problems:

$$
\begin{aligned}
& \left\{R_{p}\right\}(\{u\})=\{0\}, \\
& \left\{\tilde{R}_{p}\right\}(\{u\})=\{0\},
\end{aligned}
$$

are non linear, we use the Newton-Raphson method. At each iteration $j$ we solve the following linear system:

$$
\begin{aligned}
& {\left[\frac{\partial\{R\}}{\partial\{u\}}\right]\left(\left\{u^{j-1}\right\}\right)\{\delta u\}=-\{R\}\left(\left\{u^{j-1}\right\}\right),} \\
& \left\{u^{j}\right\}=\{\delta u\}+\left\{u^{j-1}\right\} .
\end{aligned}
$$

For $p=1$ the problem is linear. Increasing $p$ progressively, we initialise the Newton-Raphson algorithm by choosing for $\left\{u^{0}\right\}$ the displacement field computed with a smaller value of $p$.

The differentiation of formula (28) leads to the following tangent matrix:

$$
\begin{aligned}
{\left[\frac{\partial\left\{\tilde{R}_{p}\right\}}{\partial\{u\}}\right]=} & \sum_{e} \int_{V^{e}}\left(\{u\}^{T}\left[K^{e}\right]\{u\}\right)^{p-1}\left[K^{e}\right] \mathrm{d} s \\
& +2(p-1) \sum_{e} \int_{V^{e}}\left(\{u\}^{T}\left[K^{e}\right]\{u\}\right)^{p-2}\left[K^{e}\right]\{u\}\left(\left[K^{e}\right]\{u\}\right)^{T} \mathrm{~d} s
\end{aligned}
$$

which we can use directly while the differentiation of formula (27) leads to a full tangent matrix:

$$
\begin{aligned}
{\left[\frac{\partial\left\{R_{p}\right\}}{\partial\{u\}]=}\right.} & \mathcal{V}\left(\sum_{e} \int_{V^{e}}\left(\{u\}^{T}\left[K^{e}\right]\{u\}\right)^{p} \mathrm{~d} s\right)^{1 / p-1} \sum_{e} \int_{V^{e}}\left(\{u\}^{T}\left[K^{e}\right]\{u\}\right)^{p-1}\left[K^{e}\right] \mathrm{d} s \\
& +2(p-1) \mathcal{V}\left(\sum_{e} \int_{V^{e}}\left(\{u\}^{T}\left[K^{e}\right]\{u\}\right)^{p} \mathrm{~d} s\right)^{1 / p-1} \\
& \times \sum_{e} \int_{V^{e}}\left(\{u\}^{T}\left[K^{e}\right]\{u\}\right)^{p-2}\left[K^{e}\right]\{u\}\left(\left[K^{e}\right]\{u\}\right)^{T} \mathrm{~d} s \\
& +2(1-p) \mathcal{V}\left(\sum_{e} \int_{V^{e}}\left(\{u\}^{T}\left[K^{e}\right]\{u\}\right)^{p} \mathrm{~d} s\right)^{1 / p-2} \\
& \times\left(\sum_{e} \int_{V^{e}}\left(\{u\}^{T}\left[K^{e}\right]\{u\}\right)^{p-1}\left[K^{e}\right]\{u\} \mathrm{d} s\right)\left(\sum_{e} \int_{V^{e}}\left(\{u\}^{T}\left[K^{e}\right]\{u\}\right)^{p-1}\left[K^{e}\right]\{u\} \mathrm{d} s\right)^{T} .
\end{aligned}
$$

As using such a full tangent matrix is numerically expensive, it is better to replace it by a truncated one. This can be done by neglecting the last term in (31). We have tested both methods and their performances are similar. 


\section{Comparison with an analytical solution}

\subsection{An analytical optimal design}

In order to test our numerical procedure, we construct in this section a non-trivial analytical solution: we consider the case of an elastic material with a vanishing Poisson's coefficient. Then $D_{i j k \ell}=2 \mu \delta_{i k} \delta_{j \ell}$ and the constraint for the displacement field $u$ takes the simple form:

$$
\varepsilon_{u}: D: \varepsilon_{u}=2 \mu \varepsilon_{u}: \varepsilon_{u} \leqslant 1 \text {. }
$$

Using adapted units, we consider $2 \mu=1$. Then, in support of the optimal design $\{x, h(x)>0\}$ where the constraint is active (the inequality is actually an equality), the strain tensor has to verify:

$$
\varepsilon_{11}^{2}+\varepsilon_{22}^{2}+2 \varepsilon_{12}^{2}=1
$$

where $\varepsilon_{i j}$ denote the components of $\varepsilon_{u}$ in an orthogonal basis $\left(x_{1}, x_{2}\right)$.

On the other hand, as $\varepsilon_{u}$ is a symmetric part of a gradient, it has to verify the compatibility condition:

$$
\frac{\partial^{2} \varepsilon_{11}}{\partial x_{2}^{2}}+\frac{\partial^{2} \varepsilon_{22}}{\partial x_{1}^{2}}-2 \frac{\partial^{2} \varepsilon_{12}}{\partial x_{1} \partial x_{2}}=0
$$

Finally, $u$ must correspond to the equilibrium displacement field of the optimal design. In a domain free from external forces, this condition states that there exists a scalar field $h(x)$ such that $\operatorname{div}\left(h \varepsilon_{u}\right)=0$, i.e.:

$$
h \operatorname{div}\left(\varepsilon_{u}\right)+\operatorname{grad}(h) \cdot \varepsilon_{u}=0,
$$

or

$$
\operatorname{div}\left(\varepsilon_{u}\right)+\operatorname{grad}(\ln (h)) \cdot \varepsilon_{u}=0 .
$$

The existence of such a field $h$ is assured, when $\varepsilon_{u}$ is invertible, if $\varepsilon_{u}$ satisfies:

$$
\operatorname{rot}\left(\varepsilon_{u}^{-1} \cdot \operatorname{div}\left(\varepsilon_{u}\right)\right)=0 .
$$

It is difficult to describe the general properties of the solutions of equations (33), (34) and (36), but it is easy to find particular non-trivial solutions.

Example 1: Let us determine, for instance, a solution $\varepsilon_{u}$ diagonal in the Cartesian coordinate system $\left(x_{1}, x_{2}\right)$, i.e. such that $\varepsilon_{12}(x)=0$ everywhere. Then equations (33), (34) and (36) become:

$$
\varepsilon_{11}^{2}+\varepsilon_{22}^{2}=1, \quad \frac{\partial^{2} \varepsilon_{11}}{\partial x_{2}^{2}}+\frac{\partial^{2} \varepsilon_{22}}{\partial x_{1}^{2}}=0, \quad \frac{\partial^{2}}{\partial x_{1} \partial x_{2}}\left(\ln \left(\frac{\varepsilon_{22}}{\varepsilon_{11}}\right)\right)=0
$$

a solution of which is:

$$
\varepsilon_{11}=-\left(1+\left(\frac{x_{2}}{x_{1}}\right)^{4}\right)^{-1 / 2}, \quad \varepsilon_{22}=-\left(1+\left(\frac{x_{1}}{x_{2}}\right)^{4}\right)^{-1 / 2} .
$$


The thickness $h$ of the optimal plate is then given, up to a multiplicative constant, by equation (35). We have:

$$
h(x)=\gamma \sqrt{x_{1}^{-4}+x_{2}^{-4}} .
$$

Let us assume now that the plate is contained in the square $\Omega:=[a, b]^{2}$ with $0<a<b$. It is easy to determine the external density of forces $F$ which are applied on the boundary of the domain. Indeed the equilibrium condition reads $F=h \varepsilon_{u} \cdot n$, where $n$ denotes the external normal to the boundary $\partial \Omega$ of $\Omega$. For the considered domain, $F$ is parallel to $n: F=-p n$. The plate is therefore subjected to the non-constant pressure field $p$ :

$$
\begin{aligned}
& p(x)=x_{2}^{-2} \quad \text { along the vertical edges }\{a\} \times[a, b] \text { and }\{b\} \times[a, b], \\
& p(x)=x_{1}^{-2} \quad \text { along the horizontal edges }[a, b] \times\{a\} \text { and }[a, b] \times\{b\} .
\end{aligned}
$$

The design given by (39) is the optimal design of a plate contained in the square $\Omega$ and subjected to the pressure field given by (40), the multiplicative constant $\gamma$ being determined by the volume constraint. The associated strain field is given by (38).

Example 2: It is also possible to obtain solutions with cylindrical symmetry by assuming that the strain tensor is diagonal in the natural basis $\left(e_{r}, e_{\theta}\right)$ associated with the polar coordinates $(r, \theta)$. Such solutions (for which $\left.\varepsilon_{r \theta}=0\right)$ correspond to more realistic loads than (40). Their computation is more intricate than in the previous example and we only give the results. The parametric description of the optimal designs has the form:

$$
\begin{aligned}
& r=a \mathrm{e}^{t / 2}|\cos (t)-\sin (t)|^{1 / 2}, \\
& h=b \mathrm{e}^{-t}(\cos (t))^{-1}
\end{aligned}
$$

where the constants $a$ and $b$ are determined by the loading. Indeed, if we consider the annulus $\left\{r_{1}<r<r_{2}\right\}$ subjected to an internal constant pressure $p_{1}$ and an external constant pressure $p_{2}$, the quantity:

$$
p:=h \varepsilon_{r r}=b \mathrm{e}^{-t}
$$

coincides with $p_{1}$ and $p_{2}$ for $r=r_{1}$ and $r=r_{2}$ respectively. This enables us to determine the constants $a$ and $b$ and the values $t_{1}$ and $t_{2}$ of the parameter $t$ which correspond to $r=r_{1}$ and $r=r_{2}$. It is remarkable that this determination is only possible if the ratio $p_{2} / p_{1}$ does not exceed critical values $\left(p_{2} / p_{1} \in\left[k_{1}, k_{2}\right]\right)$ which depend on the geometrical parameter $r_{2} / r_{1}$ (for instance, when $r_{2} / r_{1}$ is very large, $\left[k_{1}, k_{2}\right] \simeq[0.1,2.2]$ ). Outside of this interval, the optimal design is not entirely made of a regular 2-D plate: part of the mass concentrates along the circle $\left\{r=r_{1}\right\}$.

\subsection{Numerical confrontation}

In order to validate the numerical procedure presented in section 3, we compare its results with the analytical solutions. Let us consider the square domain $\Omega:=[a, b]^{2}$ submitted to the boundary forces described by (40) (see figure 1). Then the optimal design is a plate the thickness of which is given by (39).

We compute the finite element solution for increasing values of the parameter $p$. Generally we use the increment 2 for $p$. We stop at the maximum value before the Newton's method diverges. In order to check the numerical scheme, we study three different regular finite element grids with quadratic interpolation $(20 \times 20$, $40 \times 40,80 \times 80)$. 
Analysing the relative difference between the numerical and analytical solutions at every point of the domain (cf. figure 2a), we find that it is lower than $1 \%$. We can conclude that the numerical scheme presented here is efficient for solving the problem (12). The scheme's convergence is the same in both formulations (27) or (28). For a given value of $p$, we initialise the Newton's scheme by the solution obtained previously for a smaller value of $p$. Beginning with the value $p=1$ (which corresponds to a linear elastic problem) assures the convergence of the scheme. Many iterations (about 20) are needed to cross the first non-linearity $(p=3)$. Afterward, for $p>3$, the convergence is obtained within five iterations.

Analysing the $L^{2}$-norm of the difference between the numerical and analytical solutions, we observe a good agreement, which improves as $p$ increases (cf. figure $2 b$ ). Even when $p$ is small (about 10), the design obtained is close to the exact solution: it is pertinent to compute first a solution on a large mesh for a rough design, then to refine the mesh in order to obtain the best precision. Indeed, the mesh quality governs the precision and the maximal value of the parameter $p$ we can reach.

We have also considered the annulus $\left\{r_{1}<r<r_{2}\right\}$ subjected to the pressures given by (42) (when their ratio does not exceed the critical values). Again we get a very good agreement with the analytical solution (41).

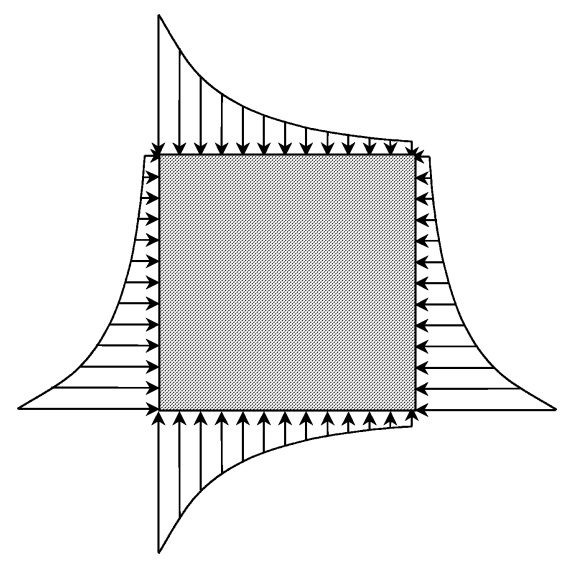

Figure 1. Boundary conditions for the numerical test.
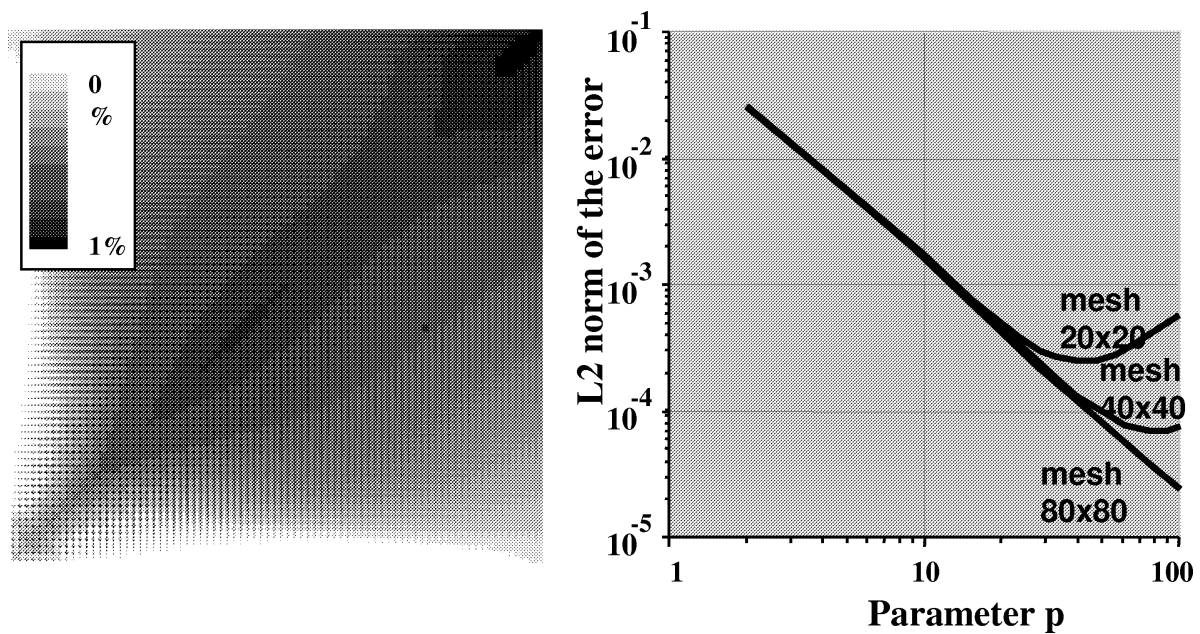

Figure 2. Difference between analytical and numerical solutions. 


\section{Topology of optimal solutions}

The goal of topological optimisation is to obtain structures, the topology of which has not been postulated a priori.

What can we say about the topology of the optimal design obtained using a fictitious material approach? Essentially, we are limited to conjectures: first, it seems reasonable, and our numerical results show, that the support of the structure (the location of non vanishing thickness) is bounded. We explored every equilibrium system of three point forces (cf. figure 3). These numerical experiments have been computed with the same relatively rough mesh and a moderate value of $p(p=10)$ : the design is not quite precise but qualitatively pertinent. The gray scale used in the figure corresponds to the thickness of the plate. The domain in which the optimal design has been sought is much larger than the support of the obtained designs. Thus, these designs are not limited by any geometrical constraint. As a matter of fact, the optimal design seems to be included in a disk containing the support of the forces. Here circles seem to play a central role for a still unknown reason. See figure 4 in which we consider a system of vertical forces applied at three points of an horizontal line: the optimal design is a disk with variable thickness. This figure can be compared to figure 2.7, p. 106 of (Bendsoe, 1995 ) in which, in a different context and under geometrical constraints, circles also appear.

Secondly, if connected, the structure seems to be simply connected. The restriction to connected structures is essential as many examples can be found of non connected optimal structures. On the other hand, this conjecture does not hold when the design domain is subjected to some geometrical constraint. For instance, we consider in figure 5 the case of three symmetrical forces acting on the wedges of an equilateral triangle. The thickness of the optimal design is small at the center of the structure but does not vanish (figure 6 represents the thickness
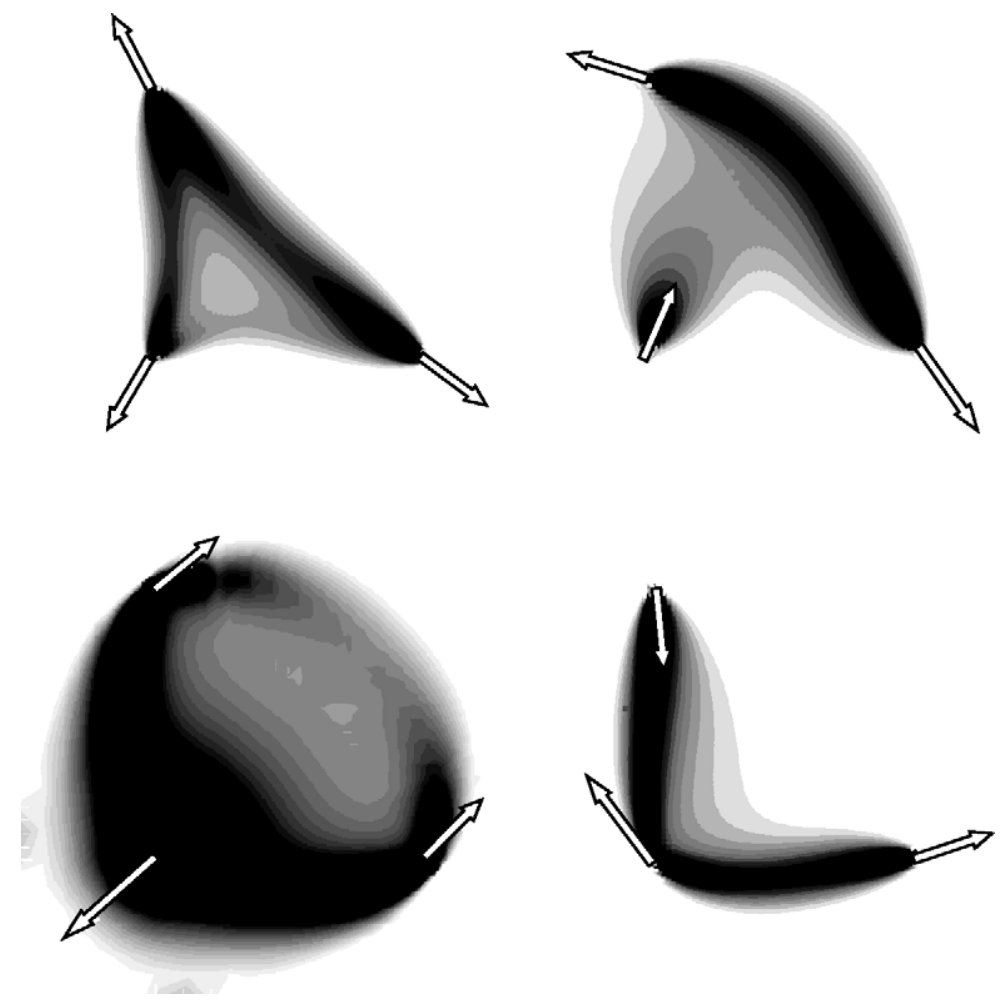

Figure 3. Optimal designs for some systems of three forces. 


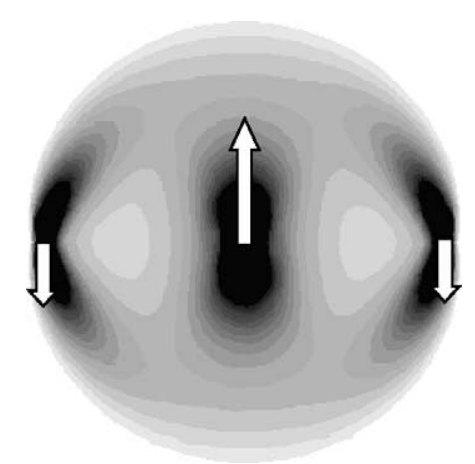

Figure 4. Optimal design for a particular system of three forces.

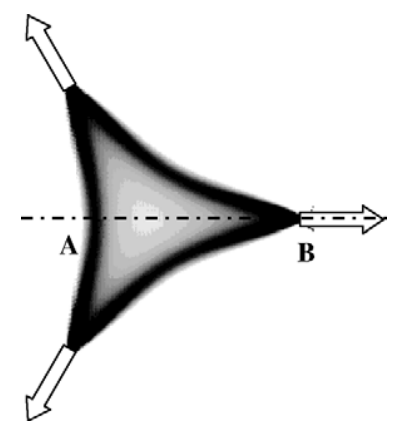

Figure 5. Optimal design for the system of three symmetrical forces.

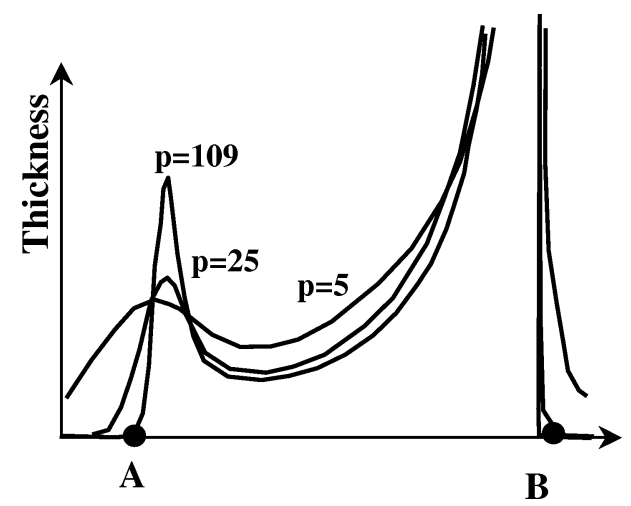

Figure 6. Thickness along the axis of symmetry.

of the design along one of its axis of symmetry obtained for different values of the parameter $p$ ). This fact is numerically clear: we verified that this minimal thickness converges to a positive value when refining the mesh and increasing the parameter $p$. Moreover this phenomenon is not an artefact of our regularisation procedure: indeed, the previous comparison between numerical and analytical solutions has showed that the numerical solutions are sufficiently accurate. Finally, we notice that, outside of the optimal design, the numerical solutions tend to zero without any ambiguity.

We explored different values for the Poisson coefficient: the optimal design depends slightly on this coefficient, but there is not any qualitative difference. Note that the triangle made by three elastic 2-D bars 
(or any other equivalent truss structure) which is an optimal design for the Michell criterion cannot be optimal for our minimisation problem. Indeed, the optimal design we obtain has a smaller compliance. This fact has been rigorously proved in (Bouchitté and Buttazzo, 2001).

We conjecture that the structure is simply connected. Up till now, we can only prove that there is no circular hole in the optimal structure: if $(h, u)$ is a regular solution of problem (12) in a domain $\Omega$ containing the disk $B(0, R)$, then it is not possible for $h$ to vanish inside a disk $B$ of radius $R_{0}$ and to be positive in its vicinity (for all $x$ in $\left.\left\{x, R_{0}<\|x\|<R_{1} \leqslant R\right\}\right)$. Indeed, as the field $\varepsilon_{u}$ is assumed to be regular, it verifies the equilibrium condition $\operatorname{div}\left(h \varepsilon_{u}\right)=0$ in the whole disk $B\left(0, R_{1}\right)$, and at each point of the boundary $\partial B$ of $B$ we have:

$$
\varepsilon_{u} \cdot n=0,
$$

$n$ denoting the external normal of $\partial B$.

The optimality condition, $\left\|\varepsilon_{u}\right\|^{2}=1$ where $h>0$, yields to:

$$
\tau \cdot \varepsilon_{u} \cdot \tau=1 \quad \text { on } \partial B
$$

( $\tau$ denoting a unit vector tangent to $\partial B$ ) and

$$
\left\|\varepsilon_{u}\right\|^{2} \leqslant 1 \quad \text { in } B .
$$

To prove that there is no field $u$ in $B$ verifying these two conditions, let us consider the following minimisation problem:

$$
\inf \left\{\int_{B}\left\|\varepsilon_{u}(x)\right\|^{2} \mathrm{~d} x, u \in \mathcal{M}\right\}
$$

where $\mathcal{M}$ denotes the space of those functions of the usual Sobolev space $H^{1}(B)$ such that $\left.u\right|_{\partial B} \in H^{1}(\partial B)$ and $\tau \cdot \varepsilon_{u} \cdot \tau=1$ on $\partial B$. This is a linear elastic equilibrium problem. The boundary condition is not usual but it has been proved in (Bouchitté et al., 1997-b) that this minimisation problem is well-posed. Therefore, there exists a unique solution $u_{0}$, which due to the symmetry of $B$, is radial. Then, it is easy to verify that $u_{0}(x)=x$ and the infimum of (45) is $\int_{B} 2 \mathrm{~d} x$. The mean value of $\left\|\varepsilon_{u}\right\|^{2}$ is larger or equal to 2 for every $u$ in $\mathcal{M}$ : no element of $\mathcal{M}$ can verify condition (44).

\section{Final remarks}

In this study we show how shape optimisation and locking materials are closely linked. First, this enables us to have mechanic intuition of optimal designs, but overall this leads to a classical numerical approach. We only have to search for the equilibrium configuration of a strongly non-linear elastic medium. The optimisation problem we considered is very particular. Many practical applications need a more sophisticated model: one should replace the fictitious energy (2) by an energy resulting from homogenisation theory; one should optimise the stiffness under multiple loads; one should consider materials with different behaviors; one should discuss the case of mass concentration along domains of lower dimension. All these variants lead to significant difficulties. Our problem is pertinent for optimisation of 2-D structures, it raises some central questions and enables us to test some conjectures. Our numerical experiments show that, as expected, the optimal structures are bounded. To our knowledge, this quite intuitive fact is not proved even for the Michell optimisation criterion. Our experiments show also, less expectedly, that the optimal structure is simply connected. This makes a fundamental difference between Michell trusses and fictitious optimal designs. Before a penalisation step, 
optimal designs obtained through homogenisation seem to present the same feature (compare figures 1 and 2 or 6 and 7 in (Allaire et al., 1997) and figures 2.17 and 2.21 in (Rossi, 1996)).

\section{References}

Allaire, G., Bonnetier, E., Francfort, G., Jouve, F., 1997. Shape optimisation by the homogenisation method. Numer. Math. 76 (1), $27-68$.

Allaire, G., Kohn, R.V., 1993. Optimal design for minimum weight and compliance in plane stress using extremal microstructure. Eur. J. Mech. A/Solids 12 (6), 839-878.

Aubin, J.P., Ekeland, I., 1984. Applied Nonlinear Analysis. Wiley, New York.

Aubry S., 1999. Etude théorique et numérique de quelques problèmes d'optimisation de formes à l'aide de méthodes d'homogénéisation, Thèse de doctorat de l'université Paris 6.

Batoz, J.L., Dhatt, G., 1995. Modélisation des Structures par Éléments Finis 1. Hermes, Paris.

Bendsoe, M.P., 1995. Optimisation of Structural Topology, Shape and Material. Springer-Verlag, Berlin.

Ben-Tal, A., Bendsoe, M.P., 1993. A new method for optimal truss topology design. SIAM J. Optimisation 3 (2), $322-358$.

Bouchitté, G., Buttazzo, G., 2001. Characterisation of optimal shapes and masses through Monge-Kantorovich equation. J. Eur. Math. Soc., to appear in.

Bouchitté, G., Buttazzo, G., Seppecher, P., 1997-a. Shape optimisation solutions via Monge-Kantorovich equation. C. R. Acad. Sci. Paris Sér. I 324, $1185-1191$.

Bouchitté, G., Buttazzo, G., Seppecher, P., 1997-b. Energies with respect to a measure and application to low dimensional structures. Calc. Var. 5, $37-54$.

Demengel, F., Suquet, P., 1986. On locking materials. Acta Appl. Math. 6, 185-211.

Duysinx P., 1996. Optimisation topologique: du milieu continu à la structure élastique, Thèse de doctorat de l'université de Liège.

Lagache, J.M., 1981. Treillis de volume minimal dans une région donnée. J. Méc. 20 (3), 415-448.

Michell, A.G.M., 1904. The limits of economy of material in frame structures. Phil. Mag. 6 (8), 589-597.

Petersson, J., 1999. A finite element analysis of optimal variable thickness sheets. SIAM J. Numer. Anal. 36 (6), 1759-1778.

Rossi J.M., 1996. Application de m'ethodes d'homog'en'eisation 'a l'étude numérique de problèmes d'optimisation de structures, Thèse de doctorat de l'université de Provence.

Rozvany, G.I.N., 1989. Structural Design via Optimality Criteria. Kluwer, Dordrecht.

Strang, G., Kohn, R.V., 1983. Hencky-Prandtl nets and constrained Michell trusses. Computer Methods in Applied Mechanics and Engineering 36, 207-222.

Zowe, J., Kocvara, M., Bendsoe, M.P., 1997. Free material optimisation via mathematical programming. Mathematical Programming 79, $445-466$. 